\title{
PRODUÇÃO FÍSICA E FINANCEIRA DOS PROCEDIMENTOS CIRÚRGICOS EM HOSPITAIS SOB GESTÃO MUNICIPAL EM RIBEIRÃO PRETO-SP ${ }^{1}$
}

\author{
Lucieli Dias Pedreschi Chaves ${ }^{2}$, Maria Luiza Anselmi, Claudia Benedita dos Santos ${ }^{4}$
}

\begin{abstract}
${ }^{1}$ Projeto de pesquisa financiado pela Fundação de Apoio Pesquisa Estado São Paulo, processo No 2006/00938-5.
${ }^{2}$ Doutora em Enfermagem. Professor Doutor do Departamento de Enfermagem Geral e Especializado da Escola de Enfermagem de Ribeirão Preto (EERP) da Universidade de São Paulo (USP). São Paulo, Brasil. E-mail: dpchaves@eerp.usp.br

${ }^{3}$ Doutora em Enfermagem. Professor Associado do Departamento de Enfermagem Geral e Especializado da EERP/USP. São Paulo, Brasil. E-mail: anselmi@eerp.usp.br

${ }^{4}$ Doutor em Estatística. Professor Associado do Departamento de Materno Infantil e Saúde Pública da EERP/USP. São Paulo, Brasil. E-mail: cbsantos@eerp.usp.br
\end{abstract}

\begin{abstract}
RESUMO: Estudo exploratório com objetivo descrever a produção física e financeira dos procedimentos cirúrgicos, nas internações hospitalares remuneradas pela Secretaria Municipal de Saúde de Ribeirão Preto, segundo prestador, período 1996-2003. A coleta de dados utilizou a pesquisa documental em banco de dados oficiais da Secretaria Municipal de Saúde sobre internações em cinco hospitais conveniados ao Sistema Único de Saúde. No período houve incremento de 73,1\% na produção física e de 121,6\% nos recursos financeiros, concentrados nas subespecialidades de ortopedia/traumatologia, gastroenterologia e cirurgias do sistema cardiovascular/linfático. Constatamos a especialização de hospitais em algumas subespecialidades cirúrgicas. A variação na produção evidencia que subespecialidades de maior incorporação tecnológica e melhor remuneração apresentam incremento. O estudo não apresenta estimativas para os anos subseqüentes, $\mathrm{o}$ comportamento das diferentes subespecialidades ou diferentes prestadores hospitalares do município, entretanto incremento na produção física e financeira dos procedimentos cirúrgicos, no município estudado, justifica uma análise crítica do gestor do sistema.

DESCRITORES: Hospital. Hospitalização. Sistema Único de Saúde. Recursos em saúde. Sistemas de informação hospitalar.

\section{PHYSICAL AND FINANCIAL TERMS OF THE SURGICAL PROCEDURES CARRIED OUT IN MUNICIPALLY MANAGED HOSPITALS IN RIBEIRÃO PRETO-SP, BRAZIL}

\begin{abstract}
This exploratory study aimed to describe the physical and financial terms of surgical procedures in hospitalizations from 1996 to 2003 covered by the Municipal Health Secretary in Ribeirão Preto, São Paulo, Brazil, according to providers. Data collection used documental research in official Municipal Health Secretary databases about hospitalizations in five hospitals associated to the Brazilian Federal Health Care System. During the period there was a 73.1\% increase in procedure production and a $121.6 \%$ increase in financial resources, mostly in orthopedics/trauma, gastroenterology, and cardiovascular/lymphatic system surgeries. We note hospital specialization in some surgical sub-specializations. Variation among production evidences increases in sub-specializations involving higher use of technologies and better remuneration. This study does not present estimates for subsequent years, behavior of the different subspecialties, or the different hospital service providers in the city. However, the significant increase in the physical and financial terms of surgical procedures justifies critical analysis from the system manager.
\end{abstract}

DESCRIPTORS: Hospital. Hospitalization. Single Health System. Health resources. Hospital information systems.

\section{PRODUCCIÓN FÍSICA Y FINANCIERA DE LOS PROCEDIMIENTOS QUIRÚRGICOS REALIZADOS EN LOS HOSPITALES MUNICIPALES DE LA CIUDAD DE RIBEIRÃO PRETO-SP}

RESUMEN: Este estudio exploratorio tuvo como objetivo describir la producción material y financiera de los procedimientos quirúrgicos realizados en las hospitalizaciones remuneradas por la Secretaría Municipal de Salud de Ribeirão Preto, según un prestador de servicios, de 1996 a 2003. Para la recolección de los datos se utilizó la investigación documental en bases de datos oficiales de la Secretaría Municipal de Salud sobre hospitalizaciones en cinco hospitales con convenio con el Sistema Único de Salud. En el período estudiado hubo un aumento del 73,1\% en el número de hospitalizaciones y 121,6\% en los recursos financieros, especialmente en las subespecializaciones de ortopedia/ traumatología, gastroenterología y cirugías del sistema cardiovascular y linfático. Se constató la especialización de hospitales en subespecialidades quirúrgicas. La variación en la producción evidencia un incremento en las subespecialidades con mayor incorporación tecnológica y mejor remuneración. El estudio no presenta estimaciones para los años siguientes, ni para el comportamiento de las diferentes subespecialidades o diferentes prestadores de servicios hospitalarios en el municipio, sin embargo, el aumento en la producción financiera y de los procedimientos quirúrgicos en el municipio estudiado, justifica el análisis crítico por el gestor del sistema.

DESCRIPTORES: Hospital. Hospitalización. Sistema Único de Salud. Recursos en salud. Sistemas de información en hospital. 


\section{INTRODUÇÃO}

A produção de internações hospitalares, traduzida no conjunto de procedimentos realizados, representa importante parcela da atenção à saúde nos municípios tanto pela complexidade de ações quanto pelo volume financeiro empregado. A variação na produção de procedimentos em cada especialidade médica merece atenção e análise crítica tanto dos gestores do sistema como de quem produz informações em saúde. Monitorar os dados das internações hospitalares pode fornecer subsídios para reordenar o sistema de saúde local e a gerência do hospital.

A necessidade de se conhecer as características dos usuários e o padrão de utilização dos serviços de saúde é primordial para o planejamento do sistema de saúde, neste sentido, o uso da epidemiologia na área de serviços de saúde tem tido crescente importância, fornecendo subsídios para as decisões dos gestores, especialmente nas suas avaliações. ${ }^{1}$

A constituição histórica de um modelo de organização de sistema de saúde hospitalocêntrico reforça a supervalorização da unidade hospitalar enquanto espaço de produção de conhecimentos e de prática de ações de saúde. Somado a isto, o atendimento a pessoas gravemente enfermas dá maior visibilidade a estas instituições, inclusive sob a perspectiva dos usuários. Entretanto, sistematicamente, vem sendo redefinido o papel dos hospitais nos sistema de saúde. Nesse sentido, no processo de gestão, particularmente no que diz respeito à produção de ações na área hospitalar, pressupõe-se objetivos para além da execução de mecanismos de controle de demanda, ou seja, espera-se processos de avaliação aprimorados, embasados em informações que subsidiem processos decisórios.

Particularmente, na gestão, o acompanhamento das internações hospitalares deve merecer atenção diferenciada tanto pelo volume e variedade de recursos materiais e humanos consumidos, quanto pela complexidade da estrutura dos serviços hospitalares e também, pela possibilidade de o gestor reestruturar a atenção básica para a prevenção e controle de doenças prevalentes nas diferentes faixas etárias em seu município e que podem ser atendidas em ambulatórios, enfim, monitorar os dados das internações hospitalares pode fornecer subsídios para reordenar o sistema de saúde local. ${ }^{2}$

O estudo das internações hospitalares faz-se prioritário no cenário atual brasileiro, caracteriza- do, pela intensa e pouco regulada incorporação tecnológica na área da saúde, resultando em crescentes gastos no setor; pelo aumento da complexidade dos casos e da elevação dos custos com internações; e, da crise dos hospitais que prestam serviços para o SUS, seja ele público, filantrópico ou privado devido à gestão não-profissional e ao desequilíbrio orçamentário. ${ }^{3}$

O Sistema de Informação Hospitalar (SIH) do Sistema Único de Saúde (SUS) possibilita a construção de indicadores que são úteis para a monitoração e avaliação da assistência à saúde, da estrutura dos serviços de saúde e da política médico-assistencial. Entretanto, sua utilização em pesquisas epidemiológicas e seu uso para traçar o perfil de morbidade das internações hospitalares ainda é incipiente. O estabelecimento de uma rotina de utilização crítica dos dados gerados por este Sistema é imprescindível para o mapeamento de demandas populacionais por serviços de saúde, assim como para o aprimoramento do próprio sistema. ${ }^{4}$

A produção de internações hospitalares, no município de Ribeirão Preto, no período de 1996-2003, sofreu considerável variação tanto nos aspectos físicos como financeiros, sendo que o crescimento financeiro foi superior ao físico. $\mathrm{O}$ incremento de produção foi diferenciado entre prestadores e entre as especialidades médicas. Evidenciou-se que o crescimento é mais acentuado na especialidade de clínica cirúrgica onde se concentram procedimentos que agregam maior tecnologia e maior valor de remuneração segundo a tabela SIH-SUS e, portanto representam, para o prestador, um segmento de interesse em razão do aporte financeiro empregado. ${ }^{5}$

Embora estes resultados se refiram ao caso de um município específico, esta situação pode também refletir o perfil de internações hospitalares de outras localidades brasileiras de grande porte populacional e pólos regionais. A especialidade de clínica cirúrgica concentra procedimentos que são altamente especializados, consomem considerável aporte de recursos financeiros, face às ações terapêutico-assistenciais realizadas que, de modo geral, só podem ser desenvolvidos na esfera de atenção hospitalar, tais constatações justificam uma análise pormenorizada das internações hospitalares nesta especialidade e a realização deste estudo.

Sob uma perspectiva ampliada de avaliação e controle na gestão local, questionamos: na especialidade de clínica cirúrgica quais procedimentos 
foram produzidos e como eles se distribuem entre os prestadores em termos físicos e financeiros, no período 1996-2003?

A resposta a esse questionamento pode contribuir para a sistematização adequada dos dados, respectiva análise e interpretação acerca da produção e gastos com procedimentos, na especialidade de clínica cirúrgica, em internações hospitalares no município fornecendo subsídios para a ordenação do sistema local de saúde centrada nas necessidades dos usuários; para a adequação da alocação e distribuição dos recursos financeiros e, conseqüentemente, para a melhoria da atenção à saúde da população.

\section{OBJETIVO}

Descrever a produção física e financeira dos procedimentos principais realizados em internações hospitalares processadas e remuneradas pela Secretaria Municipal de Saúde de Ribeirão Preto (SMS-RP) através do SIH-SUS, na especialidade de clínica cirúrgica, para o período de 1996-2003, segundo prestador no município estudado.

\section{METODOLOGIA}

\section{Tipo de pesquisa}

Trata-se de pesquisa descritiva, populacional e transversal que, a partir de dados quantitativos, descreve e detalha os procedimentos produzidos nas internações hospitalares, na especialidade de clínica cirúrgica, tomando por referência o tipo de prestador de serviço de saúde e o ano, identificando as variações nessa produção.

\section{Local de estudo}

O presente estudo foi desenvolvido em Ribeirão Preto, município situado na região nordeste do estado de São Paulo, em 2007, com população de 559.651 habitantes que chega a 1.233.785 quando considerada a população de abrangência da Direção Regional de Saúde-XIII (DRS-XIII). ${ }^{6}$

Do ponto de vista de gestão em saúde, o município está habilitado na Gestão Plena do Sistema Municipal e, nesse sentido responde pela assistência à saúde e gestão do sistema local de saúde. Quanto ao setor hospitalar, o município, conta com uma rede de 13 hospitais sendo quatro privados filantrópicos, sete privados com fins lucrativos e dois hospitais públicos estaduais ambos conveniados com o SUS, os quais apresentam um significativo contingente de leitos hospitalares e que estão sob gestão da DRS-XIII.

\section{Campo da pesquisa}

Foram considerados campo de estudo desta pesquisa cinco hospitais gerais, identificados como A, B, C, D e E, conveniados/contratados pelo SUS através da SMS-RP, a partir do seguinte critério: apresentarem ao longo do período de estudo uma demanda expressiva de procedimentos em internações na especialidade de clínica cirúrgica. A escolha do período de estudo (1996-2003) justificase porque coincide com o início da habilitação do município à gestão semiplena, quando Ribeirão Preto assumiu a responsabilidade pela gestão local do sistema de saúde, incluindo as internações nos hospitais conveniados/contratados pelo SUS e, ainda porque estudo anterior evidenciou crescimento da produção de internações na especialidade cirúrgica.

Com base nas informações contidas na ficha de cadastro de estabelecimentos de saúde, os hospitais incluídos neste estudo podem ser caracterizados como: hospitais gerais, que atendem demanda espontânea e referenciada, de pacientes do SUS e de outros convênios, nas especialidades de clínica médica, cirúrgica, obstetrícia e pediatria, em regime de atenção ambulatorial e de internação, com atividade auxiliar de ensino, sendo quatro filantrópicos e um privado, disponibilizando 594 leitos, dos quais 282 para o SUS.

\section{População}

A população de estudo foi constituída por todos os procedimentos principais nas internações hospitalares processadas/pagas pela SMS-RP, através do SIH-SUS, nos hospitais estudados, no período de 1996-2003, na especialidade de clínica cirúrgica.

Foram critérios de inclusão no estudo: os procedimentos principais das internações cirúrgicas regulados, realizados, autorizados, auditados por críticas do sistema de informação do Departamento de Informática do SUS (DATASUS), remunerados pela SMS-RP e que, portanto, atenderam as exigências preconizadas pelo SIH-SUS.

\section{Procedimentos de coleta e análise de dados}

Como técnica de coleta de dados foi utilizada a pesquisa documental cuja fonte foi um banco de dados referente às internações hospitalares, 
disponibilizado pelo DATASUS e pela SMS-RP, no qual foram coletados dados da produção mensal de Autorizações de Internação Hospitalar (AIH) pagas na especialidade de clínica cirúrgica, particularmente detalhadas quanto aos procedimentos principais realizados e o respectivo pagamento autorizado para cada prestador; em seguida, os dados foram agrupados por ano segundo as seguintes variáveis de interesse: número de procedimentos na especialidade de clínica cirúrgica para os hospitais estudados (prestador); valores monetários pagos (em reais) por prestador a cada ano.

Considerando o expressivo montante de procedimentos, estes foram agrupados em subespecialidades cirúrgicas seguindo as terminologias empregadas pelo DATASUS, a saber: cirurgia múltipla, urológica, cardiovascular-linfático, gastroenterológica, ginecológica, obstétrica, oftalmológica, otorrinolaringológica, plástica, ortopedia e traumatológica, neurológica, torácica, buco-maxilar, transplante renal.

Os dados foram digitados, armazenados em um novo banco de dados estruturado no programa Microsoft Excel e a partir deste foram elaboradas tabelas que sumarizam os achados. A análise dos dados foi realizada a partir do referencial teórico adotado, qual seja a reorganização do sistema de saúde no município na perspectiva do SUS.

O trabalho foi desenvolvido de modo a garantir o cumprimento dos preceitos da Resolução No 196/96 do Conselho Federal de Saúde sobre pesquisa envolvendo seres humanos, tendo sido aprovado pelo Comitê de Ética em Pesquisa da Escola de Enfermagem de Ribeirão Preto-USP, parecer $\mathrm{N}^{\mathrm{o}} 0651 / 2006$.

\section{RESULTADOS}

Nos hospitais sob gestão municipal em Ribeirão Preto-SP, no período, 1996 a 2003 atenderam ao critério de inclusão 66.680 internações.

A produção dos procedimentos principais das internações da especialidade de clínica cirúrgica, ano a ano, de 1996 (6.407 internações) a 2003 (11.091 internações), evidencia que no período de oito anos houve um incremento global de $73,1 \%$ no total dos procedimentos produzidos. Na Figura 1 apresentamos a distribuição percentual da produção física de procedimentos principais nas internações cirúrgicas, segundo ano e subespecialidades.

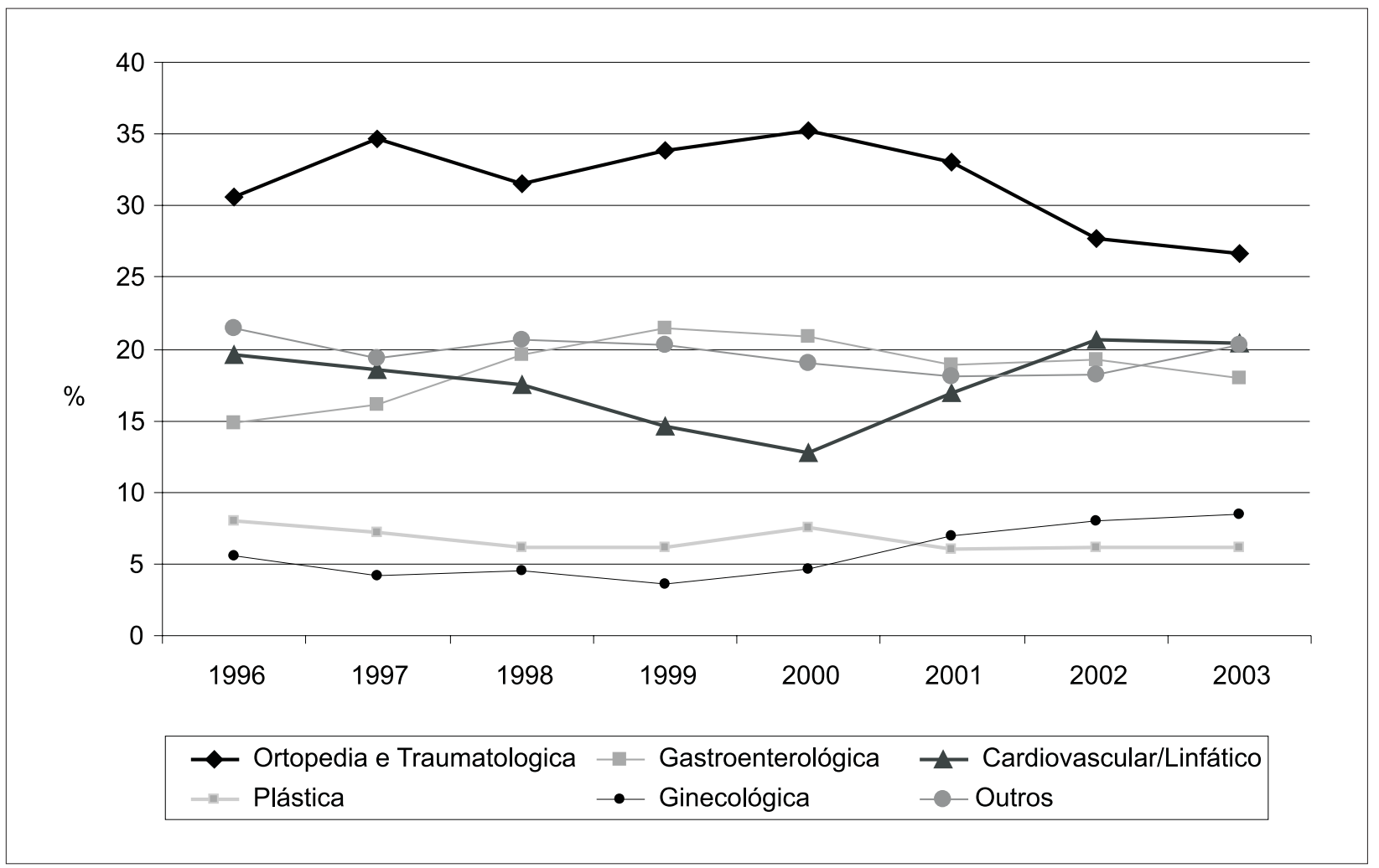

Fonte: SMS-RP, $2005^{7}$

Figura 1 - Produção física de procedimentos principais em internações cirúrgicas SUS processadas/ pagas pela SMS-RP, segundo ano e subespecialidade cirúrgica. Ribeirão Preto-SP, 1996-2003 
Quando analisadas individualmente as subespecialidades, verificamos que ortopedia/ traumatologia, gastroenterologia, cirurgias do sistema cardiovascular-linfático são responsáveis por, respectivamente, $31,3 \%, 18,8 \%$ e $17,7 \%$ da produção de 1996-2003 e, portanto, juntas concentram $67,8 \%$ dos procedimentos realizados no período todo, enquanto todas as demais subespecialidades quando somadas chegam a aproximadamente $32,2 \%$ do total de procedimentos no período.
A produção financeira referente aos procedimentos principais das internações da especialidade de clínica cirúrgica, ano a ano, de 1996 (R\$ $5.639 .501,85)$ a 2003 ( $\mathrm{R} \$ 12.500 .273,33)$, indica que no período de oito anos houve um incremento global de $121,6 \%$ nos recursos monetários empregados para pagamento de procedimentos.

Os percentuais dos valores financeiros despendidos com os procedimentos principais nas internações hospitalares nas subespecialidades da clínica cirúrgica estão apresentados na Figura 2.

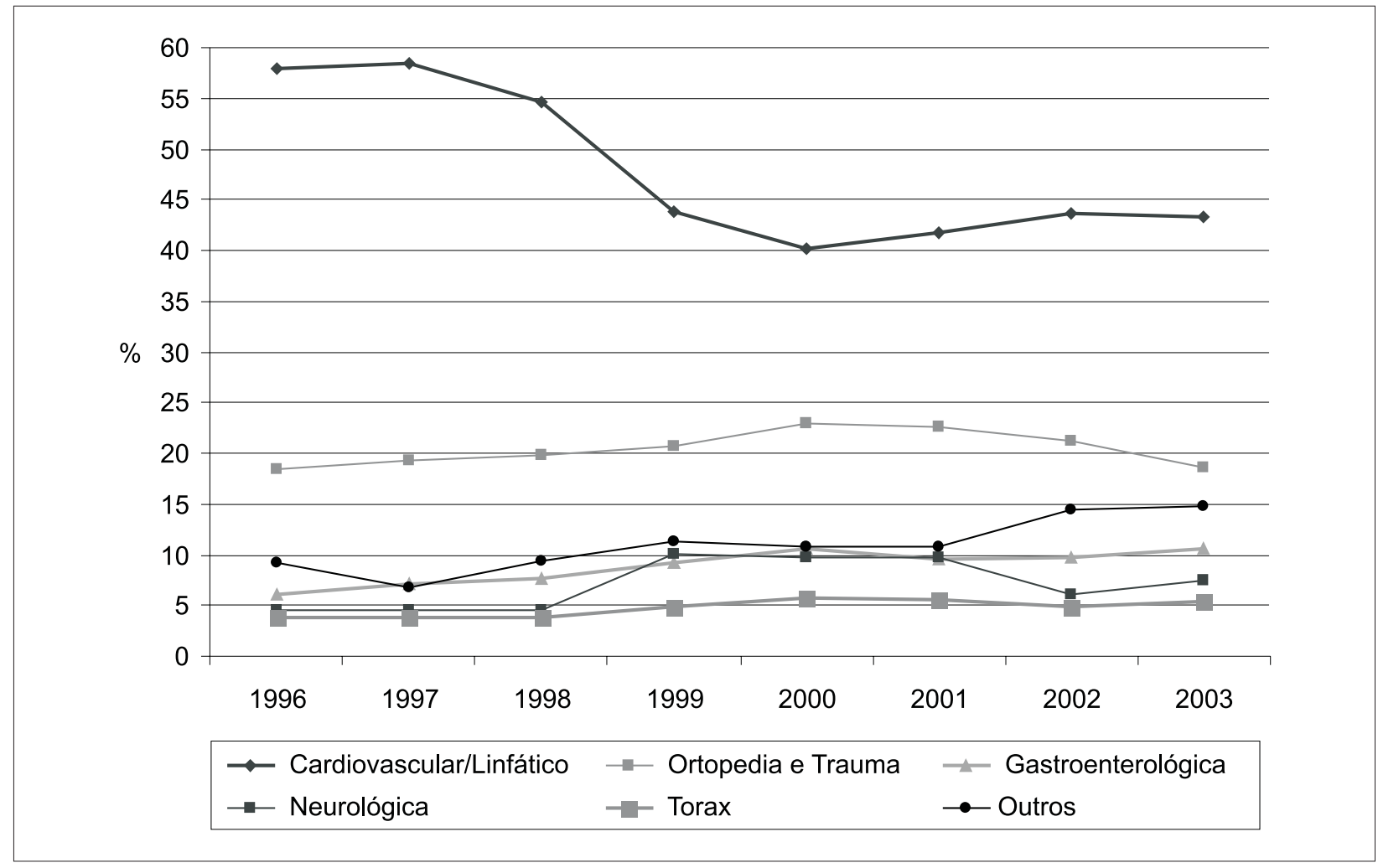

Fonte: SMS-RP, $2005^{7}$

Figura 2 - Produção financeira de procedimentos principais em internações cirúrgicas SUS processadas/pagas pela SMS-RP, segundo ano e subespecialidade cirúrgica. Ribeirão Preto-SP, 1996-2003

Os grupos de cirurgias do sistema cardiovasculares/sistema linfático, ortopedia, gastroenterologia são responsáveis por, respectivamente, $45,8 \%, 20,6 \%$ e $9,3 \%$ dos gastos no período, totalizando $75,7 \%$ dos recursos financeiros consumidos. Merece destaque o fato de as cirurgias do sistema cardiovascular-linfático, no tocante a frequência dos procedimentos, ser a terceira subespecialidade mais frequente e a primeira no montante de recursos financeiros, indicando um grupo de procedimentos cujos valores de remuneração são mais elevados em relação aos demais, uma vez que incorpora tecnologias recentes como o stent, marcapassos multisítios, dentre outros, que agregam valor ao procedimento faturado.

Os valores despendidos com a remuneração das subespecialidades de urologia, ginecologia, plástica, transplante renal, otorrinolaringologia, oftalmologia, buco-maxilar, endocrinologia e cirurgia obstétrica, totalizam aproximadamente $11,9 \%$ dos recursos financeiros consumidos no período, ou seja, um grande número de subespecialidades com baixo montante de produção e remuneração. 
Quanto ao incremento financeiro nos procedimentos em cirurgia neurológica entre os anos de 1998 e 1999 é provável que tal situação decorra da implantação de uma política diferenciada, por parte do Ministério da Saúde, para credencia- mento de serviços e de valorização financeira dos respectivos procedimentos.

Na Tabela 1, apresentamos a produção física dos procedimentos principais em clínica cirúrgica segundo prestador.

Tabela 1 - Produção física de procedimentos principais em internações cirúrgicas SUS processadas/ pagas pela SMS-RP, segundo prestador e subespecialidade cirúrgica. Ribeirão Preto-SP, 1996-2003

\begin{tabular}{|c|c|c|c|c|c|c|}
\hline \multirow{2}{*}{ Subespecialidades } & \multicolumn{5}{|c|}{ Hospital } & \multirow{2}{*}{ Total (\%) } \\
\hline & $A(\%)$ & B $(\%)$ & $\mathrm{C}(\%)$ & $\mathrm{D}(\%)$ & $E(\%)$ & \\
\hline Ortopedia e traumatologia & 3,8 & 17,5 & 35,2 & 49,7 & - & 31,3 \\
\hline Gastroenterológica & 3,4 & 29,1 & 14,1 & 15,4 & 0,1 & 18,8 \\
\hline Cardiovascular-linfático & 71,4 & 18,2 & 18,4 & 11,5 & - & 17,7 \\
\hline Plástica & 0,8 & 5,4 & 8,4 & 7,3 & 0,1 & 6,6 \\
\hline Ginecológica & 0,3 & 4,4 & 7,2 & 2,0 & 67,3 & 6,1 \\
\hline Neurológica & 0,7 & 12,7 & 2,5 & 1,7 & - & 5,6 \\
\hline Tórax & 3,9 & 5,4 & 9,0 & 0,8 & - & 5,1 \\
\hline Urológica & 9,2 & 4,2 & 2,4 & 1,8 & 32,5 & 3,9 \\
\hline Otorrinolaringologista & 0,1 & 0,8 & 1,1 & 7,5 & - & 2,7 \\
\hline Múltipla & 0,6 & 1,4 & 0,9 & 1,7 & - & 1,2 \\
\hline Oftalmológica & - & 0,5 & 0,4 & 0,4 & - & 0,4 \\
\hline Buco maxilar & - & 0,4 & 0,3 & 0,1 & - & 0,3 \\
\hline Transplante renal & 5,7 & - & - & - & - & 0,2 \\
\hline Endocrinologia & - & - & 0,1 & 0,1 & - & 0,1 \\
\hline Obstétrica & 0,1 & - & - & - & - & - \\
\hline Total & 100,0 & 100,0 & 100,0 & 100,0 & 100,0 & 100,0 \\
\hline
\end{tabular}

Fonte: SMS-RP, $2005^{7}$

A Tabela 1 evidencia a vocação e a especificidade de cada instituição em diferentes subespecialidades cirúrgicas. No Hospital A, os procedimentos principais nas subespecialiadades de cirurgia do sistema cardiovascular-linfático, urologia e transplante renal, correspondem a $71,4 \%, 9,2 \%, 5,7 \%$, respectivamente. O Hospital $\mathrm{B}$ apresenta expressiva produção em cirurgias gastroenterológicas, cirurgia do sistema cardiovascular-linfático e ortopedia/traumatologia que totalizam respectivamente, $29,1 \%, 18,2 \%$, $17,5 \%$. No hospital C a produção concentra-se em cirurgias de ortopedia/traumatologia, sistema cardiovascular-linfático e gastroenterológica que respondem por $35,2 \%, 18,4 \%, 14,1 \%$ das cirurgias realizadas neste período. Para o Hospital D as cirurgias em ortopedia/traumatologia correspondem a 49,7\%. O Hospital E desponta como o prestador cuja produção se concentra em duas especialidades, ginecologia e urologia, que, respectivamente correspondem a $67,3 \%$ e $32,5 \%$ indicando uma acentuada especialização do hospital nestas áreas, que juntas somam $99,8 \%$ da produção.

Na Tabela 2, apresentamos a produção financeira dos procedimentos principais nas subespecialidades cirúrgicas segundo prestador. 
Tabela 2 - Produção financeira de procedimentos principais em internações cirúrgicas SUS processadas/ pagas pela SMS-RP, segundo prestador e subespecialidade cirúrgica. Ribeirão Preto-SP, 1996-2003

\begin{tabular}{lrrrrrr}
\hline \multirow{2}{*}{ Subespecialidades } & \multicolumn{5}{c}{ Hospital } & Total (\%) \\
\cline { 2 - 5 } Cardiovascular-linfático & $\mathrm{A}(\%)$ & $\mathrm{B}(\%)$ & $\mathrm{C}(\%)$ & $\mathrm{D}(\%)$ & $\mathrm{E}(\%)$ & \\
Ortopedia e traumatologica & 65,4 & 51,2 & 48,8 & 10,5 & - & 45,8 \\
Gastroenterológica & 0,5 & 10,8 & 25,1 & 58,4 & - & 20,6 \\
Neurológica & 0,5 & 10,9 & 7,7 & 15,8 & 0,1 & 9,2 \\
Tórax & 0,2 & 15,2 & 1,7 & 3,4 & - & 7,5 \\
Urológica & 1,3 & 6,4 & 6,5 & 1,2 & - & 5,0 \\
Ginecológica & 17,4 & 1,3 & 1,3 & 0,7 & 15,9 & 3,4 \\
Plástica & - & 1,1 & 3,0 & 1,1 & 84 & 2,1 \\
Transplante renal & - & 1,1 & 4,0 & 3,4 & - & 2,1 \\
Múltipla & 14,5 & 0,1 & - & - & - & 1,9 \\
Otorrinolaringologista & 0,2 & 1,3 & 1,3 & 2,4 & - & 1,6 \\
Oftalmológica & - & 0,2 & 0,3 & 2,8 & - & 0,6 \\
Buco maxilar & - & 0,2 & 0,1 & 0,1 & - & 0,1 \\
Endocrinologia & - & 0,2 & 0,1 & 0,1 & - & 0,1 \\
Obstétrica & - & - & 0,1 & 0,1 & - & - \\
\hline Total & $\mathbf{1 0 0 , 0}$ & $\mathbf{1 0 0 , 0}$ & $\mathbf{1 0 0 , 0}$ & $\mathbf{1 0 0 , 0}$ & $\mathbf{1 0 0 , 0}$ & $\mathbf{1 0 0 , 0}$ \\
\hline
\end{tabular}

Fonte: SMS-RP, $2005^{7}$

Para o Hospital A as cirurgias cardiovasculares/ sistema linfático $(65,4 \%)$, urológicas $(17,4 \%)$ e de transplante renal $(14,5 \%)$ concentram aproximadamente $97,3 \%$ do faturamento dos procedimentos em internações cirúrgicas. No Hospital $\mathrm{B}$, as cirurgias cardiovasculares/sistema linfático $(51,2 \%)$ e as neurológicas $(15,2 \%)$ respondem por $66,4 \%$ dos recursos financeiros percebidos pelos procedimentos em internações cirúrgicas. Interessante destacar que neste hospital a produção de neurocirurgias é muito superior à produção dos demais hospitais no período. Para o Hospital C as cirurgias cardiovasculares/sistema linfático e ortopedia/traumatologia respondem juntas por $73,9 \%$ da produção financeira das internações cirúrgicas. A subespecialidade de ortopedia/ traumatologia representa, no Hospital D, 58,4\% do faturamento dos procedimentos em internações cirúrgicas. Interessante ressaltar, como dito anteriormente, que trata-se de um hospital geral, porém evidencia especialização em uma subespecialidade de elevada incorporação tecnológica e consumo de componentes de alto custo como órteses e próteses. O Hospital E, embora seja um hospital geral, desde sua inserção no sistema local de saúde, havia um predomínio das internações em ginecologia, a qual responde por $84,9 \%$ dos valores percebidos em internações cirúrgicas.

\section{DISCUSSÃO}

A adequada utilização de recursos humanos, tecnológicos e financeiros em cada esfera de atenção, atendendo ao princípio da integralidade e da hierarquização, tem trazido à tona a discussão acerca da dimensão da participação e da respectiva parcela de responsabilidade do hospital no sistema de saúde, bem como da possibilidade de, a partir de dados da produção hospitalar, reordenar a estratégia de ação do sistema local de saúde na atenção básica.

A utilização de serviços de saúde compreende diferentes desfechos, incluindo consultas médicas preventivas e curativas, procedimentos de diagnose e terapia ambulatoriais e internações 
hospitalares. A assistência hospitalar tem sido foco de atenção devido ao seu papel fundamental nos serviços de saúde e ao seu custo elevado. ${ }^{8}$

O perfil epidemiológico é um elemento de impacto sobre o hospital. Desde o final do século XX é possível observar a diminuição da mortalidade por doenças infecto-contagiosas, o aumento da mortalidade decorrente de doenças crônicodegenerativas e causas-externas, o reaparecimento de antigas enfermidades (tuberculose, cólera, dengue) e o surgimento de doenças infecto-contagiosas emergentes (aids, hantaviroses, doenças espongiforme humana). ${ }^{9}$

Em diversos países do mundo observa-se uma redução no número de leitos hospitalares decorrente de uma política de contenção de custos, melhor gerência, aumento dos limiares da atenção ambulatorial e o incremento de novos pontos de atenção intra e extra-hospitalares. No Brasil, a municipalização teve como aspecto positivo o controle e a avaliação em nível local, que dentre outros resultados provocou o incremento dos gastos ambulatoriais, perante os hospitalares, a restrição de internações psiquiátricas de longa duração e a diminuição do número de $\mathrm{AIH}$ emitidas. ${ }^{10}$

Assim, entendemos que está ocorrendo um processo de redefinição da atenção hospitalar que acarreta uma reorganização dos sistemas locais de saúde, tal situação pode ser uma possível explicação para a variação da produção de procedimentos em algumas subespecialidades. Por exemplo, os procedimentos cirúrgicos em oftalmologia, cujo atendimento foi reordenado em outras esferas do sistema de saúde que não o hospitalar, com o incremento em cirurgias ambulatoriais provavelmente justificando a baixa frequência de internações nesta subespecialidade.

Entretanto, embora as cirurgias ambulatoriais estejam sendo valorizadas como uma estratégia viável frente às necessidades de pacientes cirúrgicos, ainda hoje os procedimentos cirúrgicos atendem a uma demanda que, em geral, não poderia ser atendida em outro nível de atenção dentro do sistema de saúde que não o hospital, sendo esta uma possível explicação para o incremento na produção de procedimentos cirúrgicos. ${ }^{11}$

Outro aspecto que merece ser destacado, em razão da possibilidade de alterar a produção de procedimentos principais em internação na clínica cirúrgica, diz respeito à resolubilidade do sistema local de saúde como um todo, uma vez que serviços capacitados a enfrentar e resolver problemas até o nível de sua competência/complexidade podem intervir nos problemas de saúde da população precocemente, possibilitando a atenção satisfatória para o problema/queixa/solicitação do usuário em outros níveis de atenção que não o hospitalar, podendo utilizar condutas e terapêuticas que minimizem a necessidade de intervenções cirúrgicas. Por exemplo, o seguimento clínico, especializado a pacientes cardíacos pode favorecer a manutenção de quadros crônicos, sob controle, por longos períodos, evitando ou minimizando complicações que requerem intervenções cirúrgicas. ${ }^{5}$

Dentre os resultados deste estudo ressaltamos a expressiva produção de procedimentos cirúrgicos cardiovasculares, área de importante incorporação de novas tecnologias (por exemplo, cirurgias cardíacas com implantação de marcapassos), evidenciando a necessidade de um acompanhamento pormenorizado dos casos, com o objetivo de analisar a ocorrência de casos agudos, a agudização de casos crônicos e a adequação/ pertinência da atenção às patologias em nível clínico, ou seja, a partir desta análise dos casos que geraram a produção destes procedimentos cirúrgicos é possível viabilizar, para este grupo específico de pacientes, propostas de intervenção, em outras esferas do sistema local de saúde.

O contexto social também interfere nas necessidades de saúde da população, neste sentido, expressiva parcela da população urbana é acometida por doenças decorrentes das chamadas causas externas, expressão da violência em suas mais diversas formas e pela magnitude dos traumas. ${ }^{12}$ Os acidentes automobilísticos, motociclísticos, ferimentos por arma de fogo e arma branca podem justificar a demanda em ortopediatraumatologia, que segundo os resultados deste estudo é a subespecialidade com maior produção de procedimentos.

O expressivo consumo monetário justifica o acompanhamento pormenorizado desta produção, articulando possíveis fatores explicativos para a ampliação de recursos financeiros despendidos com as internações bem como o monitoramento do cumprimento do contrato/convênio vigente entre gestor e prestadores de serviço hospitalar. Vários procedimentos pertencentes à especialidade de clínica cirúrgica apresentam graus de incorporação tecnológica e complexidade que implicam em remuneração financeira superior a outras especialidades. Tal fato estimula os prestadores a privilegiarem/selecionarem a realização destes procedimentos.

O avanço tecnológico, os custos crescentes 
dos serviços de atenção à saúde e a situação de escassez aguda de recursos a que estão submetidos os serviços públicos impõem aos seus dirigentes a busca por estratégias que assegurem uma utilização dos recursos existentes mais racional, considerando que a eficiência deva constituir-se em um dos objetivos das organizações. ${ }^{13}$

Quanto à alocação de recursos financeiros e a ordenação do sistema de saúde, apesar dos avanços técnicos propiciados por procedimentos de regulação do sistema, o processo de descentralização mostra-se ainda fortemente marcado por políticas clientelísticas e corporativistas de intermediação de interesses que interferem no processo regulatório. ${ }^{14}$

A rede pública está praticamente ausente na prestação de serviços de alta complexidade, sendo possível constatar que a rede privada tem quase pleno monopólio nesta área, percebendo elevados montantes financeiros pela prestação desses serviços. ${ }^{15}$

Apesar desta constatação, cada gestor deve planejar a cobertura assistencial priorizando o setor público e, na seqüência, complementar os serviços disponíveis na rede com serviços de saúde do setor privado. A integração dos serviços privados ao SUS deve seguir a mesma lógica do sistema, especialmente quanto aos princípios doutrinários e administrativos. ${ }^{5}$

O incremento da produção de procedimentos principais em determinadas subespecialidades além dos aspectos relativos à necessidade de saúde da população, à vocação, organização e estruturação de cada hospital, representa também uma possibilidade de aumentar receitas, evidenciando uma lógica administrativa dos serviços no sentido de garantir sua sustentação financeira. Questionamos esta visão por entender que o hospital pertence a uma categoria de prestadores de serviço no mercado de produção, cujo valor social e os recursos empregados, finalidades e missão são muito peculiares e, portanto não podem ser analisados/ administrados somente sob a ótica econômica, na perspectiva de compra e venda de produtos.

Neste estudo, a distribuição da produção dos procedimentos entre os prestadores indica a especialização de alguns hospitais em determinados segmentos. Tal situação além dos aspectos relativos à adequação/organização de recursos materiais, humanos e equipamentos necessários, da discussão dos prós e contras da especialização dos serviços de saúde, requer também uma análise no contexto do sistema local de saúde, da inserção deste hospital e sua responsabilidade no atendimento às demandas de saúde da população. No âmbito da gestão faz-se necessário utilizar critérios de ordenação/regulação baseados nas demandas, em necessidades de saúde da população e não apenas considerando a oferta de procedimentos feita pelos prestadores.

\section{CONSIDERAÇÕES FINAIS}

Acreditamos que os hospitais têm papel social claramente definido, entretanto, considerando a conformação do SUS faz-se necessário discutir, planejar, programar, prever, implementar e avaliar a adequada utilização/inserção no sistema local de serviços de saúde, dimensionando recursos tecnológicos e financeiros de modo adequado a cada esfera de atenção, de forma a atender ao princípio da integralidade e da hierarquização da assistência.

Os serviços de saúde devem estar estruturados para atender demandas tão diversas, enfatizando a promoção da saúde e prevenção de agravos, a partir dos níveis de menor complexidade e, à medida que estes serviços tenham maior abrangência e resolubilidade, espera-se que os casos para atendimento hospitalar diminuam ou, ao menos, se limitem àqueles que realmente exigem atenção neste nível do sistema de saúde.

Os resultados deste estudo evidenciam que, a produção no período de 1996 a 2003, apresentou um incremento global de $73,1 \%$ nos procedimentos das subespecialidades de clínica cirúrgica e de $121,6 \%$, nos recursos financeiros empregados para remuneração destes procedimentos.

A variação na produção é diferenciada entre as subespecialidades de clínica cirúrgica indicando que aquelas de maior incorporação tecnológica e melhor remuneração apresentam incremento, quais sejam cirurgias do sistema cardiovasculares/sistema linfático, ortopedia, gastroenterologia. Focalizando a análise no desempenho físico e financeiro, de cada hospital, no período, constatamos a especialização do prestador em algumas subespecialidades cirúrgicas, entre elas ortopedia/traumatologia e cardiovascular/sistema linfático. Entendemos que além dos aspectos estruturais e organizacionais de cada hospital, é possível que interesses financeiros estejam vinculados a esta situação.

O estudo não apresenta estimativas para os anos subsequentes, o comportamento das diferentes subespecialidades ou os diferentes prestadores hospitalares do município, entretanto, diante dos 
resultados acreditamos que se justifica, por parte da gestão municipal, o acompanhamento da produção dos serviços com vistas ao seu monitoramento e o conhecimento das reais explicações para o aumento do volume financeiro utilizado particularmente, nas internações hospitalares cirúrgicas, de modo a ter uma avaliação continua deste segmento do sistema local de saúde.

\section{REFERÊNCIAS}

1. Capilheira MF. Fatores individuais associados à utilização de consultas médicas por adultos. Rev Saúde Pública. 2006 Jun-Jul; 40(3):436-43.

2. Picolo GD, Chaves LDP, Azevedo ALCS. A produção científica sobre avaliação em serviços de internação hospitalar no Brasil: revisão integrativa. Rev Eletr Enf [online]. 2009; 11(2):395-402. Disponível em: http:/ / www.fen.ufg.br/revista/v11/n2/v11n2a22.htm

3. Castro MSM. A utilização das internações hospitalares no Brasil: fatores associados, grandes usuários, reinternações e efeito da oferta de serviços sobre o uso [tese]. Rio de Janeiro (RJ): Escola Nacional de Saúde Pública; 2004.

4. Loyola Filho AI, Matos DL, Giatti L, Afradique ME, Peixoto SV, Lima-Costa MF. Causas de internações hospitalares entre idosos brasileiros no âmbito do Sistema Único de Saúde. Epidemiol. Serv Saúde. 2004 Nov-Dez; 13(4):229-38.

5. Chaves LDP. Produção de internações nos hospitais sob gestão municipal em Ribeirão Preto-SP, 1996-2003 [tese]. Ribeirão Preto (SP): Universidade de São Paulo. Escola de Enfermagem de Ribeirão Preto. Programa de Pós-Graduação em Enfermagem Fundamental; 2005.

6. Departamento de Informática do Sistema Único de Saúde. População residente por município 2007 [página da internet]. Brasília (DF): Ministério da
Saúde; 2007 [acesso 2008 Mar 23]. Disponível em: http:/ / www.datasus.gov.br

7. Relatório de produção - 2005 [Database]. Ribeirão Preto (SP): Secretaria Municipal de Saúde de Ribeirão Preto, Divisão de Avaliação, Controle e Auditoria; 2005.

8. Garbinato LG, Béria JU, Figueiredo ACL, Raymann B, Gigante LP, Palazzo LS, et al. Prevalência de internação hospitalar e fatores associados: um estudo de base populacional em um centro urbano no Sul do Brasil. Cad Saúde Pública 2007 Jan-Fev; 23(1):217-24

9. Vecina Neto G, Malik AM. Tendências na assistência hospitalar. Ciênc Saúde Coletiva 2007 Jul-Ago; 12(4):825-39.

10. Mendes EU. Os grandes dilemas do SUS. $2^{\mathrm{a}}$ ed. Salvador (BA): Casa da Qualidade Editora; 2001.

11. Chaves LDP, Anselmi ML. Produção de internações hospitalares pelo sistema único de saúde no município de Ribeirão Preto, SP. Rev Gaúcha Enferm. 2006 Dez; 27(4):583-92.

12. Rodrigues RI, Cerqueira DRC, Carvalho AX, Lobão WJA. Custo da violência para o sistema público de saúde no Brasil. Texto para discussão No1295. Brasília (DF): IPEA; 2007 [acesso 2008 Abril 28]. Disponível em: http://www.ipea.gov.br/ sites/000/2/publicacoes/tds/td_1295.pdf

13. Taveira M. Controle de custos em saúde: redução a qualquer preço ou racionalização na busca de eficácia? - elementos para discussão. Saúde em Debate. 1999 Set-Dez; 23(53): 68-80.

14. Gerschman S. A descentralização da política de saúde no final dos anos 1990. Rev Administração Pública. 2000 Jan-Fev; 14(1):47-53.

15. Borges DF, Fernandes KC. A descentralização das ações e serviços de saúde do SUS na cidade de Natal: democratização ou privatização?. Rev Administração Pública. 2002 Jul-Ago; 36(4):565-85.
Correspondência: Escola de Enfermagem de Ribeirão Preto/USP Departamento de Enfermagem Geral e Especializado Av Bandeirantes, 3900

14040-902 - Campus Universitário, Ribeirão Preto, SP, Brasil E-mail: dpchaves@eerp.usp.br
Recebido em: 15 de setembro de 2008 Aprovação final: 18 de agosto de 2009 